\title{
Review
}

\section{Cryopreservation for preservation of potato genetic resources}

\author{
Takao Niino*1) and Miriam Valle Arizaga ${ }^{1,2)}$ \\ 1) Gene Research Center, University of Tsukuba, Tsukuba, Ibaraki 305-8572, Japan \\ 2) Centro Nacional de Recursos Genéticos, INIFAP, Tepatitlán de Morelos 47600, México
}

\begin{abstract}
Cryopreservation is becoming a very important tool for the long-term storage of plant genetic resources and efficient cryopreservation protocols have been developed for a large number of plant species. Practical procedures, developed using in vitro tissue culture, can be a simple and reliable preservation option of potato genetic resources rather than maintaining by vegetative propagation in genebanks due their allogamous nature. Cryopreserved materials insure a long-term backup of field collections against loss of plant germplasm. Occurrence of genetic variation, in tissue culture cells during prolonged subcultures, can be avoided with suitable cryopreservation protocols that provide high regrowth, leading and facilitating a systematic and strategic cryo-banking of plant genetic resources. Cryopreservation protocols for potato reviewed here, can efficiently complement field and in vitro conservation, providing for preservation of genotypes difficult to preserve by other methods, wild types and other species decided as priority collections.
\end{abstract}

Key Words: cryopreservation, D cryo-plate, DMSO droplet, droplet vitrification, encapsulation vitrification, potato genetic resource, $\mathrm{V}$ cryo-plate.

\section{Introduction}

Cryopreservation is becoming an increasingly used method for the long-term storage of plant genetic resources (PGRs). Cryopreservation requires only a minimum of space and low level of maintenance. Methods for cryopreservation have been developed for a large number of plant species and further research is being conducted to enable adoption of this approach even more broadly ( $\mathrm{Li}$ and Pritchard 2009, Pritchard et al. 2013). A timely book 'Plant Cryopreservation -A Practical Guide' was published to aid in the use of cryopreservation techniques globally, for the preservation of all forms of plant biodiversity (Reed 2008). Also, an updated 'Genebank Standards for Plant Genetic Resources for Food and Agriculture' was issued from Commission on $\mathrm{Ge}-$ netic Resources for Food and Agriculture (FAO 2014 www. fao.org/docrep/meeting/027/mf804e.pdf). In this book, one chapter discusses genebank requirements for in vitro culture and cryopreservation. Thus cryopreservation techniques using in vitro shoot tips are recognized as a long-term storage tool for PGR.

Recent research on cryopreservation has focused on practical procedures for genebank storage, thereby enabling cells and meristems to be cryopreserved by direct transfer into liquid nitrogen $(\mathrm{LN})$. The development of simple and reli-

Communicated by K. Watanabe

Received September 5, 2014. Accepted October 22, 2014.

*Corresponding author (e-mail: niino.takao.fp@u.tsukuba.ac.jp) able methods for cryopreservation facilitates cryo-banking. Optimal cryopreservation conditions produced high levels of regrowth after LN storage. Potato is one of the most important food crops for food security. There are more than 4,500 varieties of Solanum tuberosum L. (Hils and Pieterse 2009). Preservation of potato genetic resources (GRs) in genebanks is mostly by vegetative propagation due their allogamous nature and many genebanks are maintaining potato GRs as field collections.

A review by Kaczmarezyk et al. (2011) discussed in detail recent advances in potato cryopreservation based on thermal, biochemical, genomic and ultrastructural analyses. There are some recent reviews of the development of potato cryopreservation protocols (Gonzalez-Amano et al. 2008, Keller et al. 2008, Wang et al. 2008). This review introduces practical and successful cryopreservation protocols of in vitro grown potato shoot tips, which have been used and are being implemented for cryo-banking in institutions around the world. In addition, here new cryopreservation techniques using aluminium plates developed by Yamamoto et al. (2011b) and Niino et al. (2013) are described and the importance of cryopreservation techniques for long-term storage are discussed.

\section{Ex situ preservation}

Ex situ preservation of PGRs is the storage of seeds or plant materials under artificial conditions to maintain their longterm viability and availability for use. Globally, genebanks 
are employing seed storage, field collections, in vitro storage (tissue culture or cryopreservation) for ex situ preservation of PGR. Storage of PGR that produce orthodox seeds, which are tolerant to low moisture content and low temperatures, at appropriate temperature and humidity, is the most convenient ex situ preservation method. Many major seed crops are included this category. However, recalcitrant seeds, which are sensitive to low moisture content and low temperatures, do not survive if they are stored under the standard storage conditions used for orthodox seeds. This category of seeds includes several important tropical and sub-tropical tree species. There is one more category recognized as intermediates between orthodox and recalcitrant seeds and known as intermediate seeds, which can tolerate combinations of desiccation and low temperature.

Field genebanks maintain living plants. Field genebanks are used for the plants which produce non-orthodox seeds or no seeds and are vegetatively propagated. Vegetatively propagated plants comprise of two types, the perennial and annual/biennial types. The former can be maintained in the field for long period without replanting but for the latter replanting is necessary annually or biennially. The preservation of these PGRs requires an adequate area of land and continuous maintenance. Vegetatively maintained PGRs are vulnerable to loss from natural disasters and damage caused by pests and diseases.

In vitro genebanks are a means to overcome the disadvantage of the field genebanks and reflect progress in plant tissue culture techniques. Preserved in vitro germplasm can be propagated and regenerated into plantlets in a sterile and pathogen free environment. In vitro genebanks are used for species with an established tissue culture system. To maintain in vitro germplasm, it should be subcultured after specific periods of time under standard culture conditions to avoid deterioration and/or contamination of materials. Several slow growth (minimal growth) methods have been established for short (3 months) to middle (3 years) term storage using low temperature, minimal nutrition, growth retardant and so on, singularly or in combination (Oka and Niino 1997). A drawback of tissue culture storage is the induction of genetic variation or mutation during prolonged subculturing. For this reason, minimal growth method is desirable for preservation of in vitro materials reduce the subculture interval. Selection of explant is also important in in vitro storage as somaclonal variation from cultured cells and callus may occur more easily compared to in vitro shoot cultures. Hence there is a preference for using shoots for in vitro storage by minimal growth method. Details regarding preservation methods can be found at: http://cropgenebank.sgrp.cgiar. org/index.php/procedures-mainmenu-243/conservationmainmenu-198.

\section{In vitro genebanks of potato}

In vitro potato collection of 1,223 accessions are maintained at $25^{\circ} \mathrm{C}$ in the National Institute of Crop Science (NICS),
Rural Development Administration (RDA), Rep. Korea (Kim personal communication 2014). Also, in the National Center for Seeds and Seedlings (NCSS), Japan, an in vitro potato collection of 130 accessions is maintained at $25^{\circ} \mathrm{C}$ and normal subculture by node or shoot is performed every 3-4 months. The frequency of subculturing can be reduced by growing the micro plants on Murashige and Skoog medium (MS medium, Murashige and Skoog 1962) supplemented with growth retardants or osmotic stress inducing polyols, incubating them under low temperature, low light intensity and varied photoperiod (Gopal and Chauhan 2010). The Leibniz Institute of Plant Genetics and Crop Plant Research (IPK), Germany maintains 2,855 potato accessions in vitro at $4{ }^{\circ} \mathrm{C}$ as microtubers. The cycle of slow growth maintenance consists of a warm phase with longday at $20^{\circ} \mathrm{C}$ for $2-3$ months, a microtuber induction phase with short-day at $9^{\circ} \mathrm{C}$ for $2-4$ months and a cold storage period, in which microtubers are stored at $4^{\circ} \mathrm{C}$ for $12-15$ months (Keller et al. 2006, Keller personal communication 2014). At the Central Potato Research Institute, Shimla, India, more than 1,500 parental lines and potato varieties are maintained in vitro on MS medium supplemented with $40 \mathrm{~g} / 1$ sucrose and $20 \mathrm{~g} / 1$ mannitol at $6-8^{\circ} \mathrm{C}$ and 16 -h photoperiod (Gopal and Chauhan 2010). At the International Potato Center (CIP), Peru, the in vitro collection consists of 4,062 accessions that are maintained under slow growth conditions. The medium used contains MS salts, $40 \mathrm{~g} / 1$ sorbitol, $20 \mathrm{~g} / 1$ sucrose, $2 \mathrm{mg} / 1$ glycine, $0.5 \mathrm{mg} / 1$ nicotinic acid, $0.5 \mathrm{mg} / 1$ pyridoxine, $0.4 \mathrm{mg} / 1$ thiamine and $8 \mathrm{~g} / \mathrm{l}$ agar. Cultures are maintained at $6-8^{\circ} \mathrm{C}$ under $22 \mu \mathrm{mol} / \mathrm{m}^{2} \mathrm{~s}$ illumination and 16-h light. This allows in vitro plantlets to be stored for approximately 2 years without sub-culturing (Panta personal communication 2014). Besides these Institutes, in vitro storage of potato GRs is conducted at many other Institutes, such as National Forestry, Crops and Livestock Research Institute, Mexico and National Institute for Agricultural Research, Chile. Almost all Institutes mentioned above have started research on cryopreservation of potato GRs as an alternate of in vitro storage.

\section{The concept of vitrification}

Cryopreservation is based on the reduction and subsequent interruption of metabolic functions of biological materials by decreasing the temperature with $\mathrm{LN}\left(-196^{\circ} \mathrm{C}\right)$, while maintaining viability. At $-196^{\circ} \mathrm{C}$, almost all the cellular metabolic activities are quiescent and the cells can be preserved in such state for a long-term. It is essential to avoid lethal intracellular freezing that occurs during rapid cooling in LN and warming in order to maintain the viability of hydrated cells and tissues (Sakai and Yoshida 1967). Cells and tissues that are to be cryopreserved in LN, need to be sufficiently dehydrated before being immersed in LN.

There are two types of liquid-solid phase transitions in aqueous solutions. (a) Ice formation is the phase transition from liquid to ice crystals, and (b) vitrification is a phase 
transition from a liquid to amorphous glass that avoids crystallization (Sakai et al. 2008). Water is very difficult to vitrify because the growth rate of crystals is very fast, even just below freezing point. However, highly concentrated cryoprotective solutions such as glycerol are very viscous and are easily supercooled below $-70^{\circ} \mathrm{C}$. This allows them to be vitrified on rapid cooling (Sakai 1997, Sakai et al. 2008). Vitrification refers to the physical process by which a highly concentrated cryoprotective solution supercools to very low temperatures and finally solidifies into a metastable glass without crystallization (Fahy et al. 1984). Vitrification had been proposed as a method for the cryopreservation of biological materials because of the potentially detrimental effects of extracellular and intracellular freezing might be avoided (Luyet 1937). Thus, vitrification is an effective freeze-avoidance mechanism for hydrated cells and tissues. As glass fills space in a tissue, it may contribute to the prevention of additional tissue collapse, solute concentration, and $\mathrm{pH}$ alteration during dehydration. Operationally, glass is expected to exhibit a lower water vapor pressure than the corresponding crystalline solid, thereby preventing further dehydration. Because glass is exceedingly viscous and stops all chemical reactions that require molecular diffusion, its formation leads to dormancy and stability over time (Burke 1986). In any cryopreservation method, whole specimens or partial parts of specimens, which are in sufficient concentration of cytosol, can vitrify by rapid cooling into LN. In the plant vitrification method, plant vitrification solution (PVS) is used which is an extremely concentrated solution (7-8 M) of cryoprotectants. The most applied PVS is PVS2 solution which contains $30 \%(\mathrm{w} / \mathrm{v})$ glycerol, $15 \%(\mathrm{w} / \mathrm{v})$ ethylene glycol, 15\% (w/v) dimethyl sulfoxide (DMSO) and $0.4 \mathrm{M}$ sucrose in basal MS medium (Matsumoto and Niino 2014, Sakai et al. 1990). This solution is supercooled below $-70^{\circ} \mathrm{C}$ and vitrified at about $-115^{\circ} \mathrm{C}$ without any detectable freezing events (Sakai et al. 1990, 1991).

\section{Cryopreservation}

Preservation of in vitro shoot tips and somatic embryos at cryogenic temperatures is considered to be a suitable alternative that can ensure the long-term security of vegetatively maintained germplasm. Once stored in LN, germplasm can be kept for apparently almost unlimited periods, and as a result cryopreservation is the most appropriate for longterm storage of base collections. Cryopreservation is often combined with tissue culture preservation for in vitro storage. Because the cryopreservation procedure is usually proceeded by tissue culture, except when preserving seeds, pollen and dormant buds.

In the vitrification method, cells and tissues must be sufficiently dehydrated with plant vitrification solution without causing injury to be capable of vitrifying upon rapid cooling into LN. High survival of in vitro grown materials is determined not only by the cryogenic protocol itself, but also by the physiological conditions of the materials to be cryopre- served. This means that some steps for acquisition dehydration tolerance or low temperature tolerance are crucial in the cryopreservation procedure. Following procedures such as preconditioning, preculture and osmoprotection (loading treatment) are vital for successful cryopreservation (Sakai et al. 2008). The materials must be in an optimal physiological and morphological state to ensure high recovery and vigorous regrowth after LN exposure (Engelmann et al. 2008, Sakai et al. 2008). In preconditioning, homogeneous specimens in terms of size, cellular composition, physiological state and growth stage, increase the chances of a positive and uniform response to treatment with loading solution (LS) and PVS2 (Niino 2006). Cold-hardening and preculture of shoot tips with sucrose-enriched media is effective for improving the post-thaw survival of some temperate and tropical species (Takagi et al. 1998), due to increased membrane stability (Kaczmarczyk et al. 2012). During preculture on sucrose-enriched medium, concentrations of sugar, starch and proline are greatly increased in the shoot tips and may enhance the stability of membranes under conditions of severe dehydration (Matsumoto and Sakai 2003). In addition, a cryoprotective or osmoprotective treatment with LS solution appears promising as a means of enhancing the dehydration tolerance of shoot tips of several species (Matsumoto 2002, Matsumoto and Niino 2014). The protective effect of this solution in cellular peri-protoplasmic spaces may be due to mitigation of the large osmotic stress from exposure to PVS2, as well as to some mechanisms that minimize the injurious membrane changes from severe dehydration (Crowe et al. 1988, Steponkus et al. 1992).

The optimal dehydration time with PVS2 or air dehydration is also important. An accurate control of the dehydration procedures and prevention of chemical toxicity injuries or excess osmotic stresses during dehydration treatment are indispensable for successful cryopreservation. Optimal dehydration time is species-specific, and may vary with the size, stage and morphological state of the shoot tips (Sakai et al. 2008). Oxidative processes involved in cryopreservation protocols may be responsible for the reduced viability of explants after LN exposure. Adding antioxidants in the cryoprotectant or the recovery media that counteract these reactions may improve recovery (Uchendu et al. 2010a, 2010b). Polyvinylpyrrolidone (PVP) and plant regulators supplementation in the recovery medium also increase regrowth significantly compared with no supplementation. PVP may be involved in adsorbing the phenolic compounds produced by dead cells (Niino et al. 2003).

\section{Practical cryo-storage}

During the last 25 years, several cryopreservation techniques have been established based on the conventional slow freezing method. These techniques such as the vitrification method, encapsulation/dehydration method and encapsulation/vitrification method, involve the steps of extraction of freezable water from the tissue cells before cooling 
(Reed 2008). As a result, vitrification of internal solutes takes place during cooling. Modified techniques have been developed which further reduce the chance for lethal icecrystal formation through the application of ultra-fast cooling and rewarming rates. These techniques are called the droplet vitrification method, V cryo-plate method and D cryo-plate method (Niino et al. 2013, Panis et al. 2005, Yamamoto et al. 2011b). Detailed descriptions of these cryopreservation protocols can be found in Reed (2008).

The current status of the main cryo-stored germplasm, apart from potato, is shown (Table 1). Seed preservation at super low temperature (by vapor or liquid phase of LN) has been successfully achieved for a wide range of crop species by the standard seed bank protocol. There are several large cryopreserved collections of orthodox seeds. In National Center for Genetic Resources Preservation (NCGRP), USA approximately $10 \%$ of the seed accessions preserved (over 37,000 accessions) have been cryopreserved. Whereas, more than 1,200 seed accessions of 50 species have been cryopreserved at the National Bureau of Plant Genetic Resources (NBPGR), India and 400 Panax ginseng seed accessions have been cryopreserved in National Agrobiodiversity Center, Rural Development Administration (NAC, RDA), Rep. Korea. At the Institute de Recherche pour le Développement (IRD), France, a cryopreserved collection of coffee germplasm (7 species, over 500 accessions) have been also stored safely in LN even though it is a non-orthodox seed (Engelmann personal communication 2014).

Some temperate woody plants can be cryopreserved by using dormant buds (Towill and Ellis 2008) and this cryo- preservation method is called 'Cryopreservation of dormant buds'. This method is now applied to Malus spp. (apple, Forsline et al. 1998), Morus spp. (mulberry, Niino 2000, Rao 2009) and Ulmus spp. (elm, Harvengt et al. 2004) at four different Institutes having a large scale cryo-storage infrastructure (Table 1). Cryopreservation protocol for mulberry dormant buds developed by National Institute of Agrobiological Sciences (NIAS), Japan is as follows. Mulberry branches with axillary buds are collected during the winter season when the buds are quiescent. After harvest, dormant buds with vascular tissue are removed and packed in polypropylene cryotube $(8 \mathrm{ml})$ which are then cooled down to $0^{\circ} \mathrm{C},-5^{\circ} \mathrm{C},-10^{\circ} \mathrm{C},-15^{\circ} \mathrm{C}$ and $-20^{\circ} \mathrm{C}$ at successive one day intervals as a pre-freezing dehydration process. The final day when buds are at $-20^{\circ} \mathrm{C}$, the cryotube is removed from the cooling unit and quickly transferred into the vapor phase of $\mathrm{LN}$ tank (ca. $-160^{\circ} \mathrm{C}$ ) for long-term storage (Niino 2000). The regrowth of 'Kenmochi' mulberry buds stored for 11.5 years in vapor phase of $\mathrm{LN}$ tank was $98 \%$ by tissue culture after rewarming (Fukui et al. 2011).

The large scale cryo-storage of in vitro shoot tips has been accomplished at several Institutes by optimizing cryopreservation protocols (Table 1). The International Network for the Improvement of Banana and Plantain (INIBAP) has been maintaining the Musa spp. cryo-bank collection of over 700 accessions by the droplet vitrification method (Panis et al. 2005, Panis 2008). The other crops which have been cryopreserved in cryo-banks, are Pyrus spp. (pear, Reed 1990), Rubus spp. (raspberry, Reed 1988), Manihot esculenta (cassava, Escobar et al. 1997), Allium sativum

Table 1. Current status of main cryo-storage in the world except potato germplasm

\begin{tabular}{|c|c|c|c|c|}
\hline Institute & Materials & Plants & $\begin{array}{c}\text { Cryo-storage } \\
\text { accessions (No.) }\end{array}$ & Cryopreservation methods \\
\hline NCGRP, USA & Orthodox seeds & $10 \%$ seeds of accession & over 37,000 & \multirow{4}{*}{$\begin{array}{l}\text { Desiccation, (Engelmann } \\
\text { personal communication 2014) }\end{array}$} \\
\hline NBPGR, India & Orthodox seeds & 50 species & 1,200 & \\
\hline NAB, RDA, Rep. Korea & Orthodox seeds & Panax ginseng & 400 & \\
\hline IRD, France & Non orthodox seeds & Coffea spp. & 500 & \\
\hline NCGRP, USA & Dormant buds & Malus spp. & 2,200 & \multirow{4}{*}{$\begin{array}{l}\text { Cryopreservation using } \\
\text { dormant buds (Slow freezing) }\end{array}$} \\
\hline NIAS, Japan & Dormant buds & Morus spp. & 1,236 & \\
\hline NBPGR, India & Dormant buds & Morus spp. & 329 & \\
\hline AFOCEL, France & Dormant buds & Ulmus spp. & 440 & \\
\hline NCGRP/NCGR, USA & In vitro shoot tips & Pyrus spp. & 100 & Slow freezing \\
\hline NCGRP/NCG, USA & In vitro shoot tips & Rubus spp. et al. & 57 & Slow freezing \\
\hline CIAT, Colombia & In vitro shoot tips & Manihot esculenta & 480 & Droplet vitrification \\
\hline INIBAP, Belgium & In vitro shoot tips & Musa spp. & 700 & Droplet vitrification \\
\hline NICS, RDA, Rep. Korea & Shoot from cloves & Allium sativum L. & 300 & Droplet vitrification \\
\hline IPK, Germany & In vitro shoot tips & Allium sativum $L$. & 101 & Vitrification \\
\hline IPK, Germany & In vitro shoot tips & Mentha L. & 86 & Droplet vitrification \\
\hline SARC, Japan & In vitro shoot tips & Wasabia japonica M. & 40 & Vitrification \\
\hline NIAS, Japan & In vitro shoot tips & Juncus effusus & 50 & D cryo-plate \\
\hline
\end{tabular}

These information obtained in 2nd International Symposium on Plant Cryopreservation (Aug. 2013), Fort Collins, Colorado, USA, except seeds. NCGRP (National Center for Genetic Resources Preservation); NCGR (National Clonal Germplasm Repository); NBPGR (National Bureau of Plant Genetic Resources); NAC, RDA (National Agrobiodiversity Center, Rural Development Administration); IRD (Institute de Recherche pour le Développement); NCGR (National Clonal Germplasm Repository); NIAS (National Institute of Agrobiological Sciences); AFOCEL (Association Forêt Cellulose); CIAT (International Center for Tropical Agriculture); INIBAP (International Network for the Improvement of Banana and Plantain); NICS RDA (National Institute of Crop Science, Rural Development Administration); IPK (Leibniz Institute of Plant Genetics and Crop Plant Research); SARC (Shimane Agriculture Research Center). 
Table 2. Current cryo-storage status of in vitro grown shoot tips of potato

\begin{tabular}{|c|c|c|c|c|c|c|c|}
\hline \multirow[b]{2}{*}{ Institute, country } & \multirow{2}{*}{$\begin{array}{c}\text { Total } \\
\text { accessions }\end{array}$} & \multicolumn{4}{|c|}{ Number of accessions } & \multirow{2}{*}{$\begin{array}{l}\text { Cryopreservation } \\
\text { methods }\end{array}$} & \multirow[b]{2}{*}{ Literature } \\
\hline & & $\begin{array}{c}\text { Field } \\
\text { preservation }\end{array}$ & $\begin{array}{c}\text { Seed } \\
\text { storage }\end{array}$ & $\begin{array}{l}\text { In vitro } \\
\text { storage }\end{array}$ & $\begin{array}{l}\text { Cryo- } \\
\text { storage }\end{array}$ & & \\
\hline IPK/GLKS, Germany & $\begin{array}{c}6,124 \\
(2,846)\end{array}$ & 89 & $\begin{array}{c}2,846 \\
(2,846)\end{array}$ & 2,855 & 1,436 & $\begin{array}{l}\text { DMSO droplet } \\
\text { vitrification }\end{array}$ & $\begin{array}{l}\text { Keller personal } \\
\text { communication } 2014\end{array}$ \\
\hline CIP, Peru & $\begin{array}{c}6,768 \\
(2,414) \\
\end{array}$ & 3,931 & $\begin{array}{c}6,125 \\
(2,289) \\
\end{array}$ & $\begin{array}{r}4,062 \\
(49) \\
\end{array}$ & 869 & $\begin{array}{l}\text { Droplet vitrification } \\
\& \text { Vitrification }\end{array}$ & $\begin{array}{l}\text { Ellis and Panta personal } \\
\text { communication } 2014\end{array}$ \\
\hline $\begin{array}{l}\text { Northern Region 6, USA } \\
\text { NCGRP, USA }\end{array}$ & 5,808 & & & & 247 & Droplet vitrification & $\begin{array}{l}\text { Jenderek personal } \\
\text { communication } 2014\end{array}$ \\
\hline $\begin{array}{l}\text { NICS, RDA, Rep. Korea } \\
\text { NAC, RDA, Rep. Korea }\end{array}$ & 1,223 & 670 & & 1,223 & 130 & Droplet vitrification & $\begin{array}{l}\text { Kim personal } \\
\text { communication } 2014\end{array}$ \\
\hline $\begin{array}{l}\text { NIAS, Japan } \\
\text { NCSS, Japan }\end{array}$ & 1,964 & 1,964 & & $\begin{array}{r}20 \\
130\end{array}$ & 20 & V cryo-plate & Yamamoto (2013) \\
\hline $\begin{array}{l}\text { KAES HRO, Japan } \\
\text { CAES HRO, Japan }\end{array}$ & 500 & 500 & & & 100 & $\begin{array}{l}\text { Encapsulation } \\
\text { vitrification }\end{array}$ & Hirai (2011) \\
\hline
\end{tabular}

( ) means number of wild potato accessions.

IPK (Leibniz Institute of Plant Genetics and Crop Plant Research); GLKS ( The Groß Lüsewitz Potato Collection); CIP (International Potato Center); NR6 (The US Potato Center); NCGRP (National Center for Genetic Resources Preservation); NICS RDA (National Institute of Crop Science, Rural Development Administration); NAC, RDA (National Agrobiodiversity Center, Rural Development Administration); NIAS (National Institute of Agrobiological Sciences); NCSS ((National Center of Seeds and Seedlings); KAES HRO (Kitami Agricultural Experiment Station, Hokkaido Research Organization); CAES HRO (Central Agricultural Experiment Station, Hokkaido Research Organization).

(garlic, Keller 2005, Kim et al. 2004a, 2004b), Menta L. (mint, Senula et al. 2007), Wasabia japonica (wasabi, Matsumoto et al. 1994, 1998), Juncus effuses (mat rush, Niino et al. 2013) (Table 1).

Practical cryo-banking of in vitro grown potato shoot tips

The current status of potato cryo-banks of in vitro grown shoot tips globally is shown (Table 2). IPK, Germany, and CIP, Peru, are two of the largest potato genebanks. Both institutes have been applying cryo-storage to potato and achieved large cryo-bank collections with over 1,456 and 869 accessions, respectively. The cryo-storage of the potato shoot tips have been also established at NCGRP, USA, NAC RDA, Rep. Korea, Central Agricultural Experiment Station, Hokkaido Research Organization (CAES HRO), Japan, and NIAS, Japan (Table 2). The cryopreservation methods used in these cryo-banks are DMSO droplet (IPK), droplet vitrification (CIP, NCGRP and NAC RDA), encapsulation vitrification (CAES HRO) and V cryo-plate (NIAS). With these methods, except encapsulation vitrification method, shoot tips are directly immersed in LN and in the rewarming solution on aluminum foil strips or cryo-plates. Cooling and warming rates are about $4,000-5,000^{\circ} \mathrm{C} / \mathrm{min}$ and about $3,000-4,000^{\circ} \mathrm{C} / \mathrm{min}$, respectively, resulting in little or no crystallization and high regrowth after rewarming (Niino et al. 2013). In contrast, conventional methods such as vitrification and encapsulation vitrification use capped cryotubes for immersion into $\mathrm{LN}$ and retrieval from LN. When using capped cryotubes, cooling and warming rates are about $100-200^{\circ} \mathrm{C} / \mathrm{min}$ and about $80-120^{\circ} \mathrm{C} / \mathrm{min}$, respectively, which are far less than new methods and this has a great impact on regrowth of cryopreserved materials (Niino et al. 2013).

\section{DMSO droplet in IPK}

Schäfer-Menuhr et al. (1994) developed the ultra-rapid cooling method for in vitro shoot tips of potato called the DMSO droplet method, by using aluminium foil. Shoot tips are treated with a $10 \%$ DMSO in liquid MS medium with $30 \mathrm{~g} / 1$ sucrose, $0.5 \mathrm{mg} / 1$ zeatin riboside, $0.2 \mathrm{mg} / 1 \mathrm{GA} 3$ and $0.5 \mathrm{mg} / \mathrm{l} \mathrm{IAA}$ (MSTo, Towill 1983) at room temperature (RT) for $2 \mathrm{~h}$ and frozen ultra-rapidly by direct immersion into $\mathrm{LN}$ in a $2.5 \mu \mathrm{l}$ droplet of the same solution on a small piece of aluminium foil (Schäfer-Menuhr 1996). The procedure is shown (Table 3) (Kaczmarczyk et al. 2009, 2011, Keller et al. 2008). This protocol is very simple because only $10 \%$ DMSO is used as cryoprotectant solution. The explants $(2-3 \mathrm{~mm})$ are incubated in MSTo medium overnight at $22^{\circ} \mathrm{C}$ and treated with cryoprotectant solution (10\% DMSO in MSTo medium) for 1-3 $\mathrm{h}$ at RT followed by transfer into droplets of $2.5 \mu \mathrm{l}$ cryoprotectant solution one by one on aluminium foil. Afterwards the aluminium foil is immersed directly into cryotube filled with LN. The explants are rewarmed quickly by putting aluminium foils in liquid MS medium with $30 \mathrm{~g} / \mathrm{l}$ sucrose at RT for regeneration. The shoot tips are plated on solid MSTo medium. Currently 1,436 potato accessions are stored at IPK using this protocol with a mean regeneration percentage of $46 \%$ (Kaczmarczyk et al. 2011). The DMSO droplet method is currently being improved. One improvement is the application of alternating preculture temperature for cold accumulation of shoots under 8 -h photoperiod at $21 / 8^{\circ} \mathrm{C}$ day/night temperature for 7 days. The other improvement is the adoption of solid medium for regeneration (Kaczmarczyk et al. 2008). 


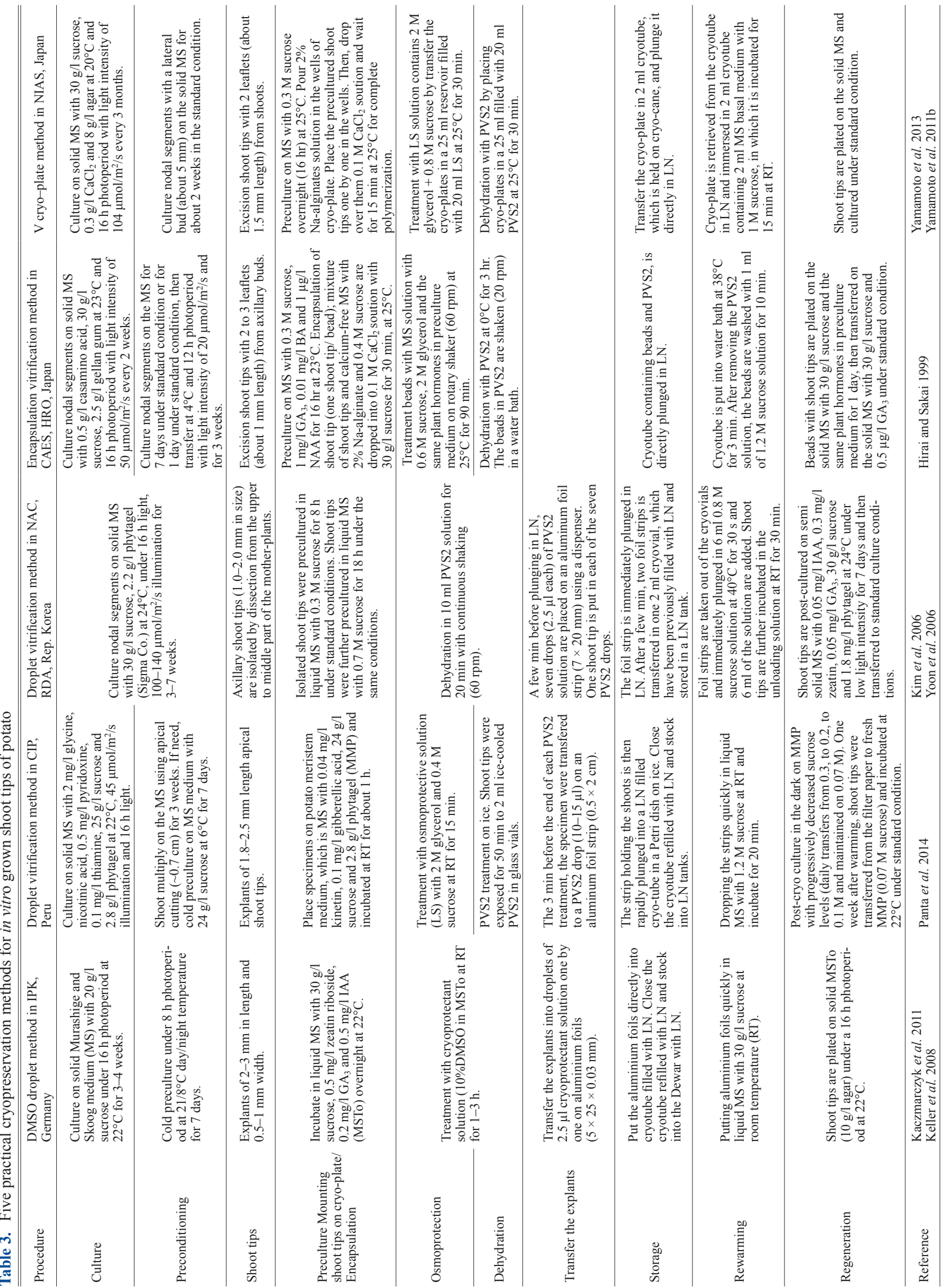


Kaczmarczyk et al. (2011) emphasized that a critical aspect in potato cryopreservation is the diverse response between different genotypes in terms of their regeneration capacities after cryopreservation. Therefore, genotypes not responsive to cryopreservation still need to be maintained using in vitro storage or field storage. In addition, certain genotypes may not even have tissue culture ability and need to be preserved as tubers (Keller et al. 2011). Finally, none of the conservation strategies (cryopreservation, in vitro storage, field storage) can be completely safe, because materials may always be lost regardless of the conservation technique.

\section{Droplet vitrification in CIP}

There are several variations of the vitrification method. The discrimination of these methods is that the standard vitrification protocol takes place in a cryotube while the droplet vitrification protocol is done on aluminium foil strips, and encapsulation vitrification adds an encapsulation step to the standard protocol (Panis 2008). The droplet vitrification method was developed in banana cryopreservation at first resulting increase in a regrowth rate of $40-50 \%$ compared to standard vitrification (Panis et al. 2005). In the droplet vitrification method, shoot tips are plated on aluminium foil during cooling and dehydrated with highly concentrated vitrification solution such as PVS2. Rewarming after cryopreservation resulted in regrowth of 46-51\% (Halmagyi et al. 2005), 8-47\% (Panta et al. 2006) and 64-94\% (Kim et al. 2006). These results confirm that regrowth ability of cryopreserved potato shoot tips is genotype-dependent as indicated by Kaczmarczyk et al. (2011). Genotype dependency was also observed by Panta et al. (2009) as the results showed that higher linoleic acid content in frost resistant genotypes of potato, are positively correlated with higher regeneration rates after cryopreservation.

The procedure of the latest droplet vitrification in CIP are shown (Table 3) (Panta et al. 2014). The shoot tips (1.3$2.5 \mathrm{~mm}$ ) are dissected from the shoots preconditioned and incubated at RT for about $1 \mathrm{~h}$ on potato meristem medium containing $24 \mathrm{~g} / 1$ sucrose, $0.04 \mathrm{mg} / 1$ kinetin and $0.1 \mathrm{mg} / 1$ gibberellic acid (MMP, Panta et al. 2014). Osmoprotection is performed by LS (MS medium with $2 \mathrm{M}$ glycerol and $0.4 \mathrm{M}$ sucrose) for $15 \mathrm{~min}$ at RT. After that shoot tips are dehydrated by PVS2 for $50 \mathrm{~min}$ at $0^{\circ} \mathrm{C}$ and $3 \mathrm{~min}$ before the end of each PVS2 treatment, the shoot tips are transferred to a PVS2 drop (10-15 $\mu \mathrm{l})$ on an aluminum foil strip $(0.5 \times$ $2 \mathrm{~cm}$ ). Then, the strip holding the shoots are rapidly immersed into a LN filled cryo-tube in a Petri dish on ice. The strips are then rewarmed quickly by dropping them in liquid MS medium with $1.2 \mathrm{M}$ sucrose at RT and incubated for 20 min for regeneration. Post-cryo culture are kept in the dark on MMP with a progressive decrease in sucrose levels (daily transfers from 0.3 , to 0.2 , to $0.1 \mathrm{M}$ and maintained on medium with $0.07 \mathrm{M}$ sucrose). One week after plating, shoot tips are transferred from the filter paper to fresh MMP $\left(0.07 \mathrm{M}\right.$ sucrose) and incubated at $22^{\circ} \mathrm{C}$ under standard conditions. This protocol was successfully applied to 4 geno- types showing different reactions to abiotic stress, which had high regrowth levels ranging from 23\% to $76 \%$ (Panta et al. 2014). The response to cryopreservation is strongly genotype and species- specific, limiting the use of the current protocols to large diverse collections such as the CIP's potato collection. To overcome this issue, it is crucial not only to optimize the protocol for different genotypes, but also to make a uniform, healthy and robust shoot tips able to tolerate cryopreservation procedures and the regeneration system. Also, it is important that the method is simple and a suitable protocol for large scale application to potato cryopreservation. In the schematic procedure, the treatment combining $15 \mathrm{~min} \mathrm{LS}$ at RT, $50 \mathrm{~min} \mathrm{PVS} 2$ at $0^{\circ} \mathrm{C}$, apical shoot tips from 3 week old mother plantlets, explant size (1.8-2.5 mm), cooling and warming on aluminium foil strips, produced positive result in the basic protocol for improving potato PVS2 vitrification (Panta et al. 2014).

\section{Droplet vitrification in NAC, RDA}

The optimization of the protocol for diverse genotypes is a prerequisite for implementing large scale cryopreservation of potato collections in genebanks. The irregular survival levels observed within different potato species could be related to many factors such as subculture conditions, size of the shoot tips and their location on the plantlet axis, sucrose concentration of the preculture medium, preculture time, dehydration, cooling and warming, and unloading step (Kim et al. 2006). The subculture conditions, light intensity, aeration and planting density significantly affected survival of cryopreserved shoot tips. Also, the subculture duration and the position of the shoot tips on the axis of the in vitro plantlets have a significant effect on survival of cryopreserved shoot tips (Yoon et al. 2006).

The procedure of droplet vitrification in NAC, RDA is shown (Table 3) (Kim et al. 2006, Yoon et al. 2006). The shoot tips $(1.0-2.0 \mathrm{~mm})$ are dissected from the subcultured shoots. The optimal duration and size of shoot tips were different by genotypes; 7 weeks and $1.5-2.0 \mathrm{~mm}$ for 'Dejima' and 5 weeks, $1.0-1.5 \mathrm{~mm}$ for 'STN13' (Yoon et al. 2006). The shoot tips are precultured with $0.3 \mathrm{M}$ sucrose for $8 \mathrm{~h}$ followed by $0.7 \mathrm{M}$ sucrose for $18 \mathrm{~h}$. The precultured shoot tips are dehydrated with PVS2 for 20 min without osmoprotection treatment. A few min before the end of each PVS2 treatment, the shoot tips are transferred to a PVS2 drop $(2.5 \mu \mathrm{l}$ each $)$ on an aluminum foil strip $(7 \times 20 \mathrm{~mm})$. Then, the strip holding the shoots are rapidly immersed into LN. For rapid warming, the strips are directly dipped in $6 \mathrm{ml} 0.8 \mathrm{M}$ sucrose solution at $40^{\circ} \mathrm{C}$ for $30 \mathrm{~s}$ and $6 \mathrm{ml}$ of the solution are added. Shoot tips are further incubated in the unloading solution at RT for $30 \mathrm{~min}$. Shoot tips are postcultured on semi solid MS with $0.05 \mathrm{mg} / 1 \mathrm{IAA}, 0.3 \mathrm{mg} / 1$ zeatin, $0.05 \mathrm{mg} / \mathrm{l} \mathrm{GA}_{3}, 30 \mathrm{~g} / 1$ sucrose and $1.8 \mathrm{mg} / \mathrm{l}$ phytagel at $24^{\circ} \mathrm{C}$ under low light intensity for 7 days and then transferred to standard culture conditions. This optimized protocol was successfully applied to 12 potato accessions, including wild species, resulting $64-94 \%$ regrowth (Kim et al. 
2006). The same protocol was applied to the Korean in vitro potato collection at NAC RDA for cryo-storage of 130 accessions.

The NCGRP USA has also adopted this protocol with slight modifications for potato cryo-storage of 247 accessions. This protocol is at the following Web site; (http://pdf. server4.org/s/solanum-tuberosum-at-ncgrp---usda-w12260. html).

\section{Encapsulation Vitrification in CAES HRO Japan}

Originally, the encapsulation-dehydration technique was developed for easy handling of a large number of meristems at the same time using the vitrification method (Matsumoto et al. 1995). Hirai and Sakai (1999) applied this method (Table 3) to various potato cultivars. In this method, uniform shoot tips from axillary shoots with 2 to 3 leaflets (approximately $1 \mathrm{~mm}$ in size) are used which are induced from nodal segments at $23^{\circ} \mathrm{C}$ for 7 days or at $4^{\circ} \mathrm{C}$ for 3 weeks. Shoot tips are precultured on MS medium supplemented with $0.3 \mathrm{M}$ sucrose, $1 \mathrm{mg} / \mathrm{l} \mathrm{GA}_{3}, 0.01 \mathrm{mg} / \mathrm{l} \mathrm{BA}$ and $1 \mu \mathrm{g} / \mathrm{l}$ NAA overnight at $23^{\circ} \mathrm{C}$. After that shoot tips are trapped within alginate gel beads (one shoot tip / one bead, diameter: 4-5 mm) with $0.4 \mathrm{M}$ sucrose. Encapsulated shoot tips are treated with a mixture of $2 \mathrm{M}$ glycerol and $0.6 \mathrm{M}$ sucrose on a rotary shaker $(60 \mathrm{rpm})$ at $25^{\circ} \mathrm{C}$ for $90 \mathrm{~min}$ to induce dehydration tolerance. They are then dehydrated with PVS2 at $0^{\circ} \mathrm{C}$ for $3 \mathrm{~h}$ in a water bath with shaking $(20 \mathrm{rpm})$. The $10-15$ beads are suspended in $1 \mathrm{ml}$ PVS2 solution per cryotube and plunged directly into LN. For regeneration, cryotube are put into a water bath at $38^{\circ} \mathrm{C}$ for $3 \mathrm{~min}$. After removing the PVS2 solution, rewarmed beads are washed with $1 \mathrm{ml}$ of $1.2 \mathrm{M}$ sucrose solution for $10 \mathrm{~min}$. Then, beads with shoot tips are plated on the solid MS medium having $3 \%$ sucrose and the same plant hormones as in preculture medium for 1 day, then transferred on the solid MS medium with $30 \mathrm{~g} / 1$ sucrose and $0.5 \mu \mathrm{g} / 1 \mathrm{GA}_{3}$ under standard condition. This protocol was successfully applied to 14 potato accessions resulting in $47-71 \%$ regrowth (Hirai and Sakai 1999). The protocol was applied to Hokkaido Research Organization (HRO) potato collection and in CAES HRO, 100 accessions have been cryopreserved in $\mathrm{LN}$ tank by using this method (Hirai 2011).

\section{V cryo-plate in NIAS}

In conventional vitrification procedures, including droplet vitrification, small size shoot tips are suspended in various solutions employed, which have to be removed and added by repeated pipetting. This often results in damage and loss of shoot tips during the course of the cryopreservation protocol. Moreover, vitrification procedures require a precise control of duration of treatment with vitrification solutions due to the narrow range of optimal treatment durations. In the droplet vitrification procedure, dehydrated shoot tips also need to be transferred on aluminium strips just before immersion in LN (Yamamoto et al. 2011b). The vitrification method using aluminium plates, named $\mathrm{V}$ cryo- plate method was developed in order to establish a simple, reproducible and reliable protocol using aluminium cryoplates. This new method is now adopted for several plant species such as strawberry (Yamamoto et al. 2011a), Dalmatian chrysanthemum (Yamamoto et al. 2011b), mint (Yamamoto et al. 2012b), mulberry (Yamamoto et al. 2012a), carnation (Sekizawa et al. 2011), mat rush (Niino et al. 2013) and sugarcane (Rafique et al. 2014). It is a userfriendly procedure and permits high cooling and warming rates of treated materials. As a result, superior regrowth is obtained after cryopreservation for the plant species which have been tested by this method.

Currently, the V cryo-plate method was successfully applied to in vitro grown potato (Yamamoto et al. 2013). The procedure is shown in Table 3. The shoots from nodal segments are cultured on solid MS medium containing $30 \mathrm{~g} / 1$ sucrose and $0.3 \mathrm{~g} / \mathrm{l} \mathrm{CaCl}_{2}$ at $20^{\circ} \mathrm{C}$ for 2 weeks. The shoot tips (about $1.5 \mathrm{~mm}$ ) are excised from the in vitro grown shoots and precultured on MS medium containing $0.3 \mathrm{M}$ sucrose at $25^{\circ} \mathrm{C}$ for overnight. The precultured shoot tips are placed on aluminium cryo-plates $(7 \mathrm{~mm} \times 37 \mathrm{~mm} \times 0.5 \mathrm{~mm})$ with ten wells (diameter $1.5 \mathrm{~mm}$, depth $0.75 \mathrm{~mm}$ ) and embedded with calcium alginate gel. Osmoprotection is performed by immersing the cryo-plates for $30 \mathrm{~min}$ at $25^{\circ} \mathrm{C}$ in $25 \mathrm{ml}$ pipetting reservoirs filled with LS (MS medium with $2 \mathrm{M}$ glycerol and $0.8 \mathrm{M}$ sucrose). For dehydration, the cryoplates are transferred and immersed in another reservoirs filled with PVS2 for $30 \mathrm{~min}$ at $25^{\circ} \mathrm{C}$. Then, the cryo-plate is transferred in an uncapped $2 \mathrm{ml}$ cryotube and directly plunged into LN. For rewarming, the cryo-plate is retrieved from the cryotube in $\mathrm{LN}$ and immersed in a $2 \mathrm{ml}$ cryotube containing $2 \mathrm{ml} \mathrm{MS}$ basal medium with $1 \mathrm{M}$ sucrose, in which it is incubated for 15 min at RT. Rewarmed shoot tips are placed on solid MS medium and cultured under standard conditions. This protocol was successfully applied to 16 cultivars and 4 wild potato accessions, resulting in high regrowth levels ranging from $93 \%$ to $100 \%$. In genebank NIAS Japan, 1,964 accessions of potato GRs are currently maintained in the field. Now, cryopreservation is a preservation option of potato GRs, as a long-term back-up system for the field collections. Currently, an air-dehydration method is being applied using aluminium cryo-plates named D cryo-plate method, which combines the encapsulationdehydration method and V cryo-plate method (Niino et al. 2013). In this method, shoot tips/buds attached to the cryoplates are dehydrated under the laminar air flow cabinet's air current after treating with LS. Air dehydration can minimize damage to specimens by avoiding the use of PVS2, using materials with comparatively higher moisture content (MC) and performing minimal excision of young leaves and/or sheaths. In in vitro mat rush buds, the D cryo-plate method overcame problems associated with sensitivity to PVS2, insufficient or excessive dehydration, damage to and loss of material during excision and manipulations (Niino et al. 2013, 2014). This method is going to be applied for in vitro grown shoots of potatoes. 


\section{Longevity and Genetic Stability}

Germplasm will survive for a long time in cryogenic storage, it is not known for exactly how long. Estimates of the actual shelf life of cryogenically stored material are critical for efficient gene-banking, but are difficult to obtain because of instrument limitations or the extended times required for measurements. The seeds of Brassica oleracea cryo-stored in $\mathrm{LN}$ for 20 years maintained their viability up to $97 \%$ after storage, but the seeds stored at $-18^{\circ} \mathrm{C}$ for 25 years had lower viability (11\%) (Walters et al. 2004). Cryogenic storage clearly prolonged shelf life of lettuce seeds with half-lives projected as $\sim 450$ and $\sim 2600$ years for fresh lettuce seeds stored in the vapor and liquid phases of liquid nitrogen, respectively (Walters et al. 2004).

Maintaining viability and genetic stability during storage is also important for cryopreserved in vitro shoot tips. After the development of vitrification methods, a few research publications appeared to suggest the exact viability and genetic stability of materials after long-term cryo-storage. Recently, Caswell and Kartha (2009) demonstrated that it was possible to cryopreserve in vitro strawberry and pea meristems in LN for 28 years. In the case of in vitro grown strawberry meristems, there was no decrease in the percentage of viable meristems stored for 8 weeks or 28 years. This result significantly extends the reported duration of successful cryopreservation of plant meristems and provides corroborating evidence to the theory that plant meristems may be cryopreserved indefinitely (Caswell and Kartha 2009). Also, for in vitro grown wasabi shoots, there was no significant differences of regrowth and morphological characteristics among 10 year cryo-storage, $2 \mathrm{~h}$ cryo-storage, treated control and control by the vitrification method (Matsumoto et al. 2013). In biochemical analyses of sinigrin, which is a chemical precursor of the mustard oil, there was no significant difference of concentration level among them. All restriction fragment length polymorphism (RAPD) fragment patterns of 10 year cryo-storage tested were identical to those of $2 \mathrm{~h}$ cryo-storage. From these results, Matsumoto et al. (2013) concluded that wasabi plants derived from shoot tips cryopreserved for 10 years by vitrification method are genetically stable. Charoensub et al. (2004) suggested that callus formation might increase the frequency of genetic variants. However, optimized cryogenic techniques with suitable conditions can provide high survival after rewarming. High survival is attributed to a lower degree of injury incurred by explants during cooling and rewarming. This indicates that a high level of survival after cryopreservation is necessary to reducing genetic changes.

In potato, many studies on genetic integrity after cryopreservation have been reported. Morphological and phenotypic, cytological and molecular comparisons were conducted revealing that plant material was genetically stable as a result of cryopreservation (Kaczmarczyk et al. 2011). Potato shoot tips cryopreserved by the DMSO droplet method and stored in LN for several years was found to have no ad- verse effect on the regeneration rates (Mix-Wagner et al. 2003). The genetic stability was also confirmed using morphological parameters, flow cytometric measurements and RFLP analyses, concluding that the cryopreservation technique may not induce somaclonal variation (SchäferMenuhr et al. 1997).

To date, many molecular and morphological study approaches for the genetic stability of cryopreserved plants had been reported. No observable significant differences have been observed in the regenerated material (Matsumoto et al. 2013). Using a biochemical approach, the diosgenin content in Dioscorea deltoidea (Dixit-Sharma et al. 2005) and the ginsenoside content in Panax ginseng (Yoshimatsu et al. 1996) were analyzed and found to be the same as those of controls. However, the possibility of some genetic changes may occur in cryopreserved plants (Kaity et al. 2008, Martin and Gonzalez-Benito 2005, Peredo et al. 2008). It is thus necessary to monitor the genetic stability of regenerated plants (true-to-type status) as a result of cryopreservation.

\section{Conclusion}

Over the last 25 years, several countries have been conserving PGR by cryopreservation. The cryopreservation technique is an effective approach for storage of plant cells, tissues, seeds and embryos. The development of new techniques for cryopreservation such as droplet vitrification, DMSO droplet and V cryo-plate methods, is facilitating the systematic and strategic cryobanking of PGRs. Cryostorage of potato germplasm is at the cutting-edge of cryopreservation research. Many experiences obtained from potato cryo-banking in IPK, CIP, NAC RDA, CAES HRO and NIAS provide insights into the storage, principles and pitfalls governing the operations of a cryo-bank. These new techniques have facilitated the cryo-banking of other plant species that are established tissue culture techniques like Musa germplasm. Reed (2008) summarized practical issues that need to be resolved before initiation of a cryo-bank. These issues include the plant materials to be preserved, storage records, storage forms, quantity to store, protocol testing, storage controls and recovery, as well as facilities and equipment. In the case of potato where huge diversity exists the genotype needs to be considered in the routine protocol of cryopreservation.

It is important to have many choices of protocol for cryopreservation, because there are many types of plant propagules and plant species to be cryopreserved. The first thing that should be done is to determine how to make materials for cryopreservation, which are not only healthy and vigorous shoots, but also at a uniform stage and size. Despite research conducted to date some genotypes remain 'recalcitrant materials'. Secondly, it is necessary to determine whether the specimens are sensitive to some chemicals such as PVS or excess dehydration. Thirdly, the unloading step and regeneration medium should be reexamined. Current 
new protocols apply rapid cooling and warming by direct immersion in LN and unloading solution. Several papers report that optimal exposure time to PVS2 shows a wide spectrum of efficiency (Sekizawa et al. 2011, Tanaka et al. 2011, Yamamoto et al. 2012a, 2012b). This means high regrowth might be obtained by rapid cooling and warming, even though the water content of specimen has not reached optimal.

Cryopreservation should be considered as a backup to field collections to insure against loss of plant germplasm (Niino et al. 2007). Priority of collections to be cryopreserved should be given to the 'at risk' plants that have an increased chance of being lost from a collection. Some minor and endangered crops have genotypes that are difficult to establish in in vitro culture. It is crucial for successful cryopreservation to develop efficient micro propagation systems. Developing tissue culture systems which allow rapid multiplication and which stimulate regrowth after retrieving samples from LN will be the next challenge for cryopreservation research.

In this review, we show practical protocols which are used in potato cryo-banks have been described. These protocols are useful techniques for cryopreservation of difficult to conserve potato genotypes, wild type potatoes, and other plant species after marginal modifications. Also, the protocols might efficiently complement one another. For example, the D cryo-plate method combines encapsulation dehydration with the $\mathrm{V}$ cryo-plate method. The physical dehydration employed in the D cryo-plate protocol might be more uniform, thus explaining the high regrowth obtained with larger shoot tips cryopreserved with the D cryo-plate (Niino et al. 2013, 2014). The D cryo-plate method may be used with larger explants, which are very sensitive or less sensitive to physical damage and cryoprotectant toxicity. To realize comprehensive cryo-storage of PGR further development of cryopreservation techniques developed are required.

\section{Acknowledgements}

We are grateful to Dr. T. Rafique for critical reading of the manuscript and to Dr. F. Engelmann for suggestions. Also, we are thankful to Drs. E.R.J. Keller, H-H. Kim, M. Jenderek, A. Panta and D. Ellis for their information related to cryopreservation. This work was supported by JST/JICA, SATREPS: Diversity Assessment and development of Sustainable Use of Mexican Genetic Resources.

\section{Literature Cited}

Burke, M.J. (1986) The glassy state and survival of anhydrous biological systems. In: Leopold, A.C. (ed.) Membrane, Metabolism and Dry Organisms. Cornell Univ. Press, Ithaca, NY, pp. 358-364.

Caswell, K.L. and K.K. Kartha (2009) Recovery of plants from pea and strawberry meristems cryopreserved for 28 years. Cryo-Lett. 30: $41-46$.

Charoensub, R., D. Hirai and A.Sakai (2004) Cryopreservation of in vitro-grown shoot tips of cassava by encapsulation-vitrification method. Cryo-Lett. 25: 51-58.

Crowe, J.H., L.M. Crowe, J.F. Carpenter, A.S. Rudolph, C.A.Wistrom, B.J. Spargo and T.J.Anchordoguy (1988) Interactions of sugars with membranes. Biochem. Biophys. Acta 947: 367-384.

Dixit-Sharma, S., S.Ahuja-Ghosh, B.B. Mandel and P.S. Srivastava (2005) Metabolic stability of plants regenerated from cryopreserved shoot tips of Dioscorea deltoidea - an endangered medicinal plant. Sci. Hortic.105: 513-517.

Engelmann, F., M-T. Gonzalez-Arnao, Y-J.Wu and R. Escobar (2008) Development of Encapsulation Dehydration. In: Reed, B. (ed.) Plant Cryopreservation: A practical Guide, Springer, New York, pp. 59-76.

Escobar, R.H., G. Mafla and W.M. Roca (1997) A methodology for recovering cassava plants from shoot tips maintained in liquid nitrogen. Plant Cell Rep. 16: 474-478.

Fahy, G.M., D.R. MacFarlane, C.A.Angell and H.T.Meryman (1984) Vitrification as an approach to cryopreservation. Cryobiology 21: 407-426.

Forsline, P.L., L.E. Towill, J.W. Waddell, C.Stushnoff, W.F.Lamboy and J.R.McFerson (1998) Recovery and longevity of cryopreserved dormant apple buds. J. Amer. Soc. Hort. Sci. 123: 365-370.

Fukui, K., K. Shirata, T. Niino and I.M. Kashif (2011) Cryopreservation of mulberry winter buds in Japan. Acta Hort. 908: 483-488.

Gonzalez-Arnao, M.T., A.Panta, W.M.Roca, R.H.Escobar and F. Engelmann (2008) Development and large scale application of cryopreservation techniques for shoot and somatic embryo cultures of tropical crops. Plant Cell Tiss. Org. Cult. 92: 1-13.

Gopal, J. and N.S. Chauhan (2010) Slow growth in vitro conservation of potato germplasm at low temperature. Potato Res. 53: 141-149.

Halmagyi,A., C. Deliu and A. Coste (2005) Plant regrowth from potato shoot tips cryopreserved by a combined vitrification-droplet method. Cryo-Lett. 26: 313-322.

Harvengt, O., A. Meier-Dinkel, E. Dumas and E. Collin (2004) Establishment of cryopreserved gene bank of European elms. Can. J. For. Res. 34: 43-55.

Hils, U. and L. Pieterse (2009) World catalogue of potato varieties 2009/10. AGRIMEDIA, Clenze.

Hirai, D. and A. Sakai (1999) Cryopreservation of in vitro-grown meristems of potato (Solanum tuberosum L.) by encapsulationvitrification. Potato Res. 42: 153-160.

Hirai, D. (2011) Gelled droplet vitrification improves recovery of cryopreserved potato germplasm. Cryo-Lett. 32: 287-296.

Kaczmarczyk,A., N.Shvachko, Y.Lupysheva, M.R.Hajirezaei and E.R.J.Keller (2008) Influence of alternating temperature preculture on cryopreservation results for potato shoot tips. Plant Cell Rep. 27: $1551-1558$.

Kaczmarczyk,A., M. Grübe and E.R.J.Keller (2009) History and development of the potato cryopreservation method and the cryopreserved collection at the IPK Gatersleben, Meeting of the WG2, COST Action 871 'Cryopreservation of Crop Species in Europe', Gatersleben, Germany. pp. 37.

Kaczmarczyk,A., V-M.Rokka and E.R.J.Keller (2011) Potato shoot tip cryopreservation. A review. Potato Res. 54: 45-79.

Kaczmarczyk, A., B. Funnekotter, A. Menon, P.Y. Phang, A.Al-Hanbali, E. Bunn and R.L. Mancera (2012) Current issues in plant cryopreservation. In: Katkov, I. (ed.) Current Frontiers in Cryobiology, InTech, Croatia, pp. 417-438.

Kaity,A., S.E.Ashmore, R.A. Drew and M.E.Dulloo (2008) Assessment of genetic and epigenetic changes following cryopreservation in papaya. Plant Cell Rep. 27: 1529-1539.

Keller, E.R.J. (2005) Improvement of cryopreservation results in garlic 
using low temperature preculture and high-quality in vitro plantlets Cryo-Lett. 26: 357-366.

Keller,E.R.J., A.Sunura, S.Leunufna and M.Grübe (2006) Slow growth storage and cryopreservation - tools to facilitate germplasm maintenance of vegetatively propagated crops in living plant collections. J. Refrigeration 29: 411-417.

Keller, E.R.J., A. Sunura and A. Kaczmarczyk (2008) Cryopreservation of Herbaceous Dicots. In: Reed, B. (ed.) Plant Cryopreservation: A practical Guide, Springer, New York, pp. 281-332.

Keller,E.R.J., A.Sunura, Ch.Zanke, M.Grübe and A.Kaczmarczyk (2011) Cryopreservation and in vitro culture-State of the art as conservation strategy for genebank. Acta Hort. 918: 99-111.

Kim,H-H., E-G.Cho, H-J.Baek, C-Y.Kim, E.R.J.Keller and F. Engelmann (2004a) Cryopreservation of garlic shoot tips by vitrification: Effect of dehydration, rewarming, unloading and regrowth conditions. Cryo-Lett. 25: 59-70.

Kim, H-H., J-B. Kim, H-J. Baek, E-G. Cho, Y-A. Chae and F. Engelmann (2004b) Evolution of DMSO concentration in garlic shoot tips during a vitrification procedure. Cryo-Lett. 25: 91-100.

Kim,H-H., J-W. Yoon, Y-E.Park, E-G.Cho, J-K. Sohn, T-S. Kim and F. Engelmann (2006) Cryopreservation of potato cultivated varieties and wild species: critical factors in droplet vitrification. CryoLett. 27: 223-234.

Li,D-Z. and H.W. Pritchard (2009) The science and economics of ex situ plant conservation. Trends Plant Sci. 14: 614-621.

Luyet, B.J. (1937) The vitrification of organic colloids and protoplasm. Biodinamica 29: 1-15.

Martin,C. and M.E. Gonzalez-Benito (2005) Survival and genetic stability of Dendranthema grandiflora Tzvelev shoot apices after cryopreservation by vitrification and encapsulation-dehydration. Cryobiology 51: 281-289.

Matsumoto,T., A. Sakai and K. Yamada (1994) Cryopreservation of in vitro-grown apical meristems of wasabi (Wasabia japonica) by vitrification and subsequent high plant regeneration. Plant Cell Rep. 13: 442-446.

Matsumoto, T., A.Sakai, C.Takahashi and K. Yamada (1995) Cryopreservation of in vitro-grown apical meristems of wasabi (Wasabia japonica) by encapsulation-vitrification method. Cryo-Lett. 16: 189-196.

Matsumoto, T., A. Sakai and Y.Nako (1998) A novel preculturing for enhancing the survival of in vitro-grown meristems of wasabi (Wasabia japonica) cooled to $-196^{\circ} \mathrm{C}$ by vitrification. Cryo-Lett. 19: 27-36.

Matsumoto, T. (2002) Herbaceous plant, Wasabi. In: Towill, L.E. and Y.P.S.Bajaj (eds.) Cryopreservation of Plant Germplasm II, Springer-Verlag, Berlin Heidelberg, pp. 180-195.

Matsumoto, T. and A. Sakai (2003) Cryopreservation of axillary shoot tips of in vitro-grown grape (Vitis) by a two-step vitrification protocol. Euphytica 131: 299-304

Matsumoto,T., T.Akihiro, S.Maki, K. Mochida, M.Kitagawa, D. Tanaka, S. Yamamoto and T. Niino (2013) Genetic stability assessment of Wasabi plants regenerated from long-term cryopreserved shoot tips using morphological, biochemical and molecular analysis. Cryo-Lett. 34: 128-136.

Matsumoto, T. and T. Niino (2014) The development of plant vitrification solution 2 and recent PVS2-based vitrification protocols. In: Reed, B.M. (ed.) Proceedings of the Second International Symposium on Plant Cryopreservation. Acta Hort. 1039: 21-28.

Mix-Wagner, G., H.M. Schumacher and R.J. Cross (2003) Recovery of potato apices after several years of storage in liquid nitrogen. CryoLett. 24: 33-41.
Murashige, T. and F. Skoog (1962) A revised medium for rapid growth and bio assays with tobacco tissue cultures. Physiol. Plant. 15: 473-497.

Niino, T. (2000) Cryopreservation of deciduous fruits and mulberry trees. In: Razdan, M.K. and E.C. Cocking (eds.) Conservation of plant genetic resources in vitro. Vol. 2, Science Publishers, New Delhi, pp. 193-221.

Niino, T., D. Tanaka, S. Ichikawa, J.Takano, S. Ivette, K. Shirata and M. Uemura (2003) Cryopreservation of in vitro-grown apical shoot tips of strawberry by vitrification. Plant Biotech. 20: 75-80.

Niino, T. (2006) Developments in PGR reservation technologies. In: Kangi, J.H. (ed.) Effective genebank management for an integrated system on sustainable conservation and utilization of plant genetic resources, RDA Korea, pp. 149-158.

Niino, T., D. Tanaka, R.R.Tantely, K. Fukui and K. Shirata (2007) Cryopreservation of basal stem buds of in vitro-grown mat rush (Juncus spp.) by vitrification. Cryo-Lett. 28: 197-206.

Niino, T., S. Yamamoto, K. Fukui, C.R.Castillo Martínez, M. Valle Arizaga, T. Matsumoto and F. Engelmann (2013) Dehydration improves cryopreservation of mat rush (Juncus decipiens Nakai) basal stem buds on cryo-plates. Cryo-Lett. 34: 549-560.

Niino, T., Wunna, K. Watanabe, N. Nohara, T. Rafique, S. Yamamoto, K. Fukui, M. Valle Arizaga, C.R. Castillo Martinez, T. Matsumoto et al. (2014) Cryopreservation of mat rush lateral buds by air dehydration using aluminum cryo-plate. Plant Biotechnol. 31: 281-287.

Oka, S. and T. Niino (1997) Long term storage of pear (Pyrus spp.) shoot cultures in vitro by minimal growth method. JARQ 31: 1-7.

Panis, B., B.Piette and R. Swennen (2005) Droplet vitrification of apical meristems: a cryopreservation protocol applicable to all Musaceae. Plant Sci. 168: 45-55.

Panis, B. (2008) Cryopreservation of Monocots. In: Reed, B. (ed.) Plant Cryopreservation: A practical Guide, Springer, New York, pp. 241-280.

Panta,A., B.Panis, C. Ynouye, B.Criel, R. Swennen and W.Roca (2006) Improvement of potato cryopreservation for the long-term conservation of Andean landraces at the International Potato Center (CIP). Cryobiology 53: 401.

Panta,A., B.Panis, D. Sanchez, P.Canepa, C. Ynouye, J.Geuns, R. Swennen, W. Roca and D. Tay (2009) Improved potato cryopreservation based on the identification of biochemical cell compounds linked to response to abiotic stress. 1st International Symposium on Cryopreservation in Horticultural Species, Leuven, Belgium, pp. 16.

Panta,A., B. Panis, C. Ynouye, R. Swennen and W. Roca (2014) Development of a PVS2 droplet vitrification method for potato cryopreservation. Cryo-Lett. 35: 255-266.

Peredo,E.L., R.Arroyo-Garcia, B.M.Reed and M.A.Revilla (2008) Genetic and epigenetic stability of cryopreserved and cold-stored hops (Humulus lupulus L.). Cryobiology 57: 234-241.

Pritchard, H.W., W. Stuppy and J. Nadarajan (2013) How many species of higher plants need conserving by cryopreservation? Abst. of 2nd International Symposium on Plant Cryopreservation. Fort Collins, Colorado, USA, pp. 15.

Rafique, T., S. Yamamoto, K. Fukui and T. Niino (2014) Cryopreservation of sugarcane by V-Cryoplate. IHC2014 Meeting Abstract.

Rao,A.A., R. Chaudhury, S.K. Malik, S. Kumar, R. Ramachandran and S.M.H. Qadri (2009) Mulberry biodiversity conservation through cryopreservation. In Vitro Cell. Dev. Biol. -Plant 45: 639-649.

Reed, B.M. (1988) Cold acclimation as a method to improve survival of cryopreserved Rubus meristems. Cryo-Lett. 9: 166-171.

Reed,B.M. (1990) Survival of in vitro-grown apical meristems of 
Pyrus following cryopreservation. HortScience 25: 111-113.

Reed, B.M. (2008) Plant Cryopreservation: A Practical Guide, Springer, New York. p. 513.

Sakai,A. and S. Yoshida (1967) Survival of plant tissue at super-low temperatures. VI. Effects of cooling and rewarming rates on survival. Plant Physiol. 42: 1695-1701.

Sakai,A., S. Kobayashi and I. Oiyama (1990) Cryopreservation of nucellar cells of navel orange (Citrus sinensis Osb. var. brasiliensis Tanaka) by vitrification. Plant Cell Rep. 9: 30-33.

Sakai,A., S. Kobayashi and I. Oiyama (1991) Survival by vitrification of nucellar cells of navel orange (Citrus sinensis var. brasiliensis Tanaka) cooled to $-196^{\circ} \mathrm{C}$. J. Plant Physiol. 137: 465-470.

Sakai,A. (1997) Potentially valuable cryogenic procedures for cryopreservation of cultured plant meristems. In: Razdan, M.K. and E.C. Cocking (eds.) Conservation of Plant Genetic Resources In Vitro, Science Publishers, USA, pp. 53-66.

Sakai,A., D. Hirai and T. Niino (2008) Development of PVS-based vitrification and encapsulation-vitrification protocols. In: Reed, B.M. (ed.) Plant Cryopreservation: A practical Guide, Springer, New York, pp. 33-58.

Schäfer-Menuhr,A., H.M. Schumacher and G.Mix-Wagner (1994) Langzeitlagerung alter Kartoffelsorten durch Kryokonservierung der Meristeme in flüssigem Stickstoff. Landbauforsch Völkenrode 44: 301-313

Schäfer-Menuhr,A. (1996) Refinement of cryopreservation techniques for potato. Final report for the period September 1, 1991-August 31, 1996. International Plant Genetic Resources Institute, Rome, Italy. pp. 1-41.

Schäfer-Menuhr,A., H.M. Schumacher and G.Mix-Wagner (1997) Long-term storage of old potato varieties by cryopreservation of shoot tips in liquid nitrogen. Plant Genet. Resour. News. 111: 1924.

Sekizawa, K., S. Yamamoto, T. Rafique, K. Fukui and T.Niino (2011) Cryopreservation of in vitro-grown shoot tips of carnation (Dianthus caryophyllus L.) by vitrification method using aluminium cryoplates. Plant Biotechnol. 28: 401-405.

Senula,A., E.R.J.Keller, T. Sanduijav and T.Yohannes (2007) Cryopreservation of cold-acclimated mint (Mentha spp.) shoot tips using a simple vitrification protocol. Cryo-Lett. 28: 1-12.

Steponkus, P.L., R. Langis and S. Fujikawa (1992) Cryopreservation of plant tissues by vitrification. In: Steponkus, P.L. (ed.) Advances in low temperature biology V.1. JAI Press, Hampton Hill, UK, pp. 1-61.

Takagi,H., N.T. Thinh and P.M.Kyesmu (1998) Cryopreservation of vegetatively propagated tropical crops by vitrification. Acta Hort. 461: 485-494.

Tanaka, D., A. Nishiuchi, T. Niino and T. Matsumoto (2011) A comparison of vitrification and droplet vitrification procedures for the cryopreservation of in vitro grown black chokeberry shoot tips. Acta
Hort. 908: 325-330.

Towill,L.E. (1983) Improved survival after cryogenic exposure of shoot tips derived from in vitro plantlet cultures of potato. Cryobiology 20: 567-573.

Towill,L.E. and D.D.Ellis (2008) Cryopreservation of dormant buds. In: Reed, B. (ed.) Plant Cryopreservation: A Practical Guide, Springer, New York, pp. 421-442.

Uchendu,E.E., S.W.Leonard, M.G.Traber and B.M.Reed (2010a) Vitamins $\mathrm{C}$ and $\mathrm{E}$ improve regrowth and reduce lipid peroxidation of blackberry shoot tips following cryopreservation. Plant Cell Rep. 29: 25-35.

Uchendu, E.E., M. Muminova, S. Gupta and B.M. Reed (2010b) Antioxidant and anti-stress compounds improve regrowth of cryopreserved Rubus shoot tips. In Vitro Cell. Dev. Biol. -Plant 46: 386393.

Walters, C., L. Wheeler and P.C. Stanwood (2004) Longevity of cryogenically stored seeds. Cryobiology 48: 229-244.

Wang, B., Z.Yin, C.Feng, S.Xiao, Y.Li and Q.Wang (2008) Cryopreservation of potato shoot tips. Fruit, Vegetable and Cereal Sci. Biotechnol. 2 (Special Issue 1): 46-53.

Yamamoto, S., K. Fukui, T. Rafique, N.I. Khan, C.R. Castillo Martinez, K. Sekizawa, T. Matsumoto and T. Niino (2011a) Cryopreservation of in vitro-grown shoot tips of strawberry by the vitrification method using aluminium cryo-plates. Plant Genetic Resources: Characterization and Utilization 10: 14-19.

Yamamoto, S., T. Rafique, W.S. Priyantha, K. Fukui, T. Matsumoto and T. Niino (2011b) Development of a cryopreservation procedure using aluminium cryo-plates. Cryo-Lett. 32: 256-265.

Yamamoto, S., T.Rafique, K. Fukui, K. Sekizawa, A.Koyama, T. Ichihashi and T. Niino (2012a) Development of an effective cryopreservation protocol using aluminium cryo-plates for in vitrogrown shoot tips of mulberries (Morus spp.) originated from the tropics and subtropics. Sanshi-Konchu Biotec. 81: 57-62.

Yamamoto, S., T. Rafique, K. Fukui, K. Sekizawa and T. Niino (2012b) V-Cryo-plate procedure as an effective protocol for cryobanks: Case study of mint cryopreservation. Cryo-Lett. 33: 12-23.

Yamamoto, S., T.Rafique, K.Fukui, C.R.Castillo Martinez and T. Niino (2013) Application of V-Cryo-plate method to in vitro shoot tips of potato varieties. Abst. of 2nd International Symposium on Plant Cryopreservation. Fort Collins, Colorado, USA, pp. 31.

Yoon, J-W., H-H.Kim, H-C.Ko, H-S. Hwang, E-S.Hong, E-G. Cho and F. Engelmann (2006) Cryopreservation of cultivated and wild potato varieties by droplet vitrification: Effect of subculture of mother-plants and of preculture of shoot tips. Cryo-Lett. 27: 211222.

Yoshimatsu, K., H. Yamaguchi and K. Shimomura (1996) Traits of Pax ginseng hairy roots after cold storage and cryopreservation. Plant Cell Rep. 15: 555-560. 